\title{
ENERGIA METABOLIZÁVEL DE INGREDIENTES PROTÉICOS DETERMINADA PELO MÉTODO DE COLETA TOTAL E POR EQUAÇÕES DE PREDIÇÃO ${ }^{1}$
}

\author{
Metabolizable energy of proteics feedstuffs, determined by the \\ total collection excreta and prediction equations
}

\author{
Márcia Cristina de Mello Zonta ${ }^{2}$, Paulo Borges Rodrigues ${ }^{3}$, Augusto Zonta ${ }^{4}$, \\ Rilke Tadeu Fonseca de Freitas ${ }^{3}$, Antonio Gilberto Bertechini ${ }^{3}$, Elias Tadeu Fialho ${ }^{5}$, Carlos Ribeiro Pereira ${ }^{6}$
}

\section{RESUMO}

Um ensaio metabólico com pintos em crescimento (método tradicional de coleta total de excretas) foi conduzido para determinar a energia metabolizável aparente corrigida (EMAn) de alguns alimentos, bem como a determinação dessa energia por equações de predição descritas na literatura. Determinou-se a EMAn de oito alimentos, sendo cinco amostras de farelos de soja de diferentes marcas comerciais e três amostras de soja integral (extrusada, tostada e micronizada). Os valores estimados pelas equações de predição foram comparados com os observados, utilizando-se a correlação de Spearman e intervalos de confiança obtidos a partir dos valores de EMAn determinados no ensaio metabólico. Os valores energéticos das amostras de farelos de soja 1, 2, 3, 4, e 5, sojas integrais extrusada, tostada e micronizada foram 2601, 2650, 2727, 2500, 2426, 3674, 3609, $4296 \mathrm{kcal} / \mathrm{kg}$ de MS, respectivamente, para a EMAn determinada com frangos de corte no ensaio metabólico. Entre as equações estudadas, as equações EMAn = -822,33 + 69,54PB - 45,26FDA + 90,81EE e EMAn $=2723,05-50,52 F D A+60,40 E E$ foram as que mais se correlacionaram $(\mathrm{P}<0,05)$ com o valor médio de EMAn observada in vivo, além de estimarem o maior número de valores energéticos dentro do intervalo de confiança calculado. A equação EMAn = 37,5PB + 46,39EE + 14,9ENN estimou todas as amostras de farelos de soja, como também a equação EMAn = 1822,76 - 99,32FB + 60,50EE + 286,73MM - 52,26AMIDO fez boas predições para as amostras soja integral, ambas correlacionadas positivamente $(\mathrm{P}<0,05)$. Diante dos resultados obtidos, conclui-se que as equações EMAn = 822,33 + 69,54PB - 45,26FDA + 90,81EE e EMAn = 2723,05 - 50,52FDA + 60,40EE são as mais indicadas para predizer valores de EMAn dos alimentos estudados e a equação EMAn = 37,5PB + 46,39EE + 14,9ENN é a mais indicada para predizer os valores energéticos dos farelos de soja.

Termos para indexação: Composição química, farelo de soja, frangos de corte, predição da energia, soja integral processada.

\section{ABSTRACT}

A metabolism assay were carried out with broilers in growth phase (traditional method of total collection of excreta) to determinate the nitrogen-corrected apparent metabolizable energy (AMEn) of some feedstuffs, as well as the determination of the energy values by prediction equations published in the national and international pappers. It was determined AMEn of eight fedstuffs, five soybean meal samples and three processed full fat samples (extruded, toasted and micronized). The estimated values were compared with observed, using the Spearman correlation and confidence intervals obtained by the metabolic assay. The energy values of soybean meals samples (1, 2, 3, 4 and 5), full fat soybean extruded, toasted and micronized were 2601, 2650, 2727, 2500, 2426, 3674, 3609, $4296 \mathrm{kcal} / \mathrm{kg}$ DM, respectively. Among the studied equations, the AMEn $=-822,33+69,54 \mathrm{CP}-$ 45,26ADF + 90,81EE and AMEn $=$ 2723,05 - 50,52ADF + 60,40EE equation correlated $(\mathrm{P}<0,05)$ with AMEn mean value observed in vivo, estimating the largest number of energy values inside of calculated confidence intervals. The equation AMEn $=37,5 \mathrm{CP}+\mathbf{4 6 , 3 9 E E}+\mathbf{1 4 , 9 N F E}$ estimated all the samples of soybean meal, as well the equation AMEn $=$ 1822,76 - 99,32CF + 60,50EE + 286,73ash $-52,26$ starch was good for full fat soybean samples, both equations was correlated $(\mathrm{P}<0,05)$. The results obtained in this assay, allow us to conclude that the equation AMEn = -822,33 + 69,54CP - 45,26ADF + 90,81EE and AMEn = 2723,05 - 50,52ADF + 60,40EE shoud be used to predict AMEn values of the studied feedstuffs. The equation AMEn $=\mathbf{3 7 , 5 C P}+\mathbf{4 6 , 3 9 E E}+$ 14,9NFE is more indicated for predict the energy values of soybean meals.

Index terms: Broiler, chemical composition, energy prediction, meal soybean, processed soybean.

(Recebido para publicação em 6 de abril de 2004 e aprovado em 19 de outubro de 2004)

1. Parte da dissertação de mestrado da primeira autora - Projeto financiado pelo CNPq.

2. Zootecnista MSc - Rua das Acácias, 184 - Jardim Eldorado - 37200-000 - Lavras, MG.

3. DS - Professores do Departamento de Zootecnia da Universidade Federal de Lavras/UFLA - Caixa Postal 3037 - $37200-000$ - Lavras, MG.

4. Estudante de mestrado DZO/UFLA.

5. PhD - DZO/UFLA

6. Bolsista do PIBIC/CNPq/UFLA. 


\section{INTRODUÇÃO}

Para se formular rações mais eficientes e atender adequadamente às exigências nutricionais dos animais, é necessário conhecer com maior precisão, entre outros, os valores energéticos dos alimentos, que podem ser determinados por meio de métodos diretos e indiretos. Os métodos diretos ou convencionais requerem a utilização de uma bomba calorimétrica e ensaios metabólicos, sendo metodologias trabalhosas, demoradas e dispendiosas e, em contrapartida, como método indireto, surgem as equações de predição, que são baseadas na composição proximal dos alimentos e obtidas rotineiramente em laboratórios é considerada uma alternativa rápida, prática e econômica na avaliação nutricional dos alimentos.

Vários pesquisadores têm desenvolvido equações para estimar a energia metabolizável por meio de sua composição proximal (NRC, 1994); porém, existem poucos relatos que venham validar tais equações em novas determinações. Janssen (1989) elaborou a Tabela Européia de Valores Energéticos de Alimentos para Aves, na qual apresenta uma série de equações de predição dos valores de EMAn para vários grupos de alimentos, fundamentado na composição química ou nos coeficientes de digestibilidade dos nutrientes (extrato etéreo, proteína bruta e extratos não nitrogenados). Rodrigues (2000) e Rodrigues et al. (2002) estimaram equações para predizer os valores energéticos da soja e subprodutos, e ressaltaram que o uso de equações com duas a quatro variáveis podem ser usadas com maior facilidade, já que necessitam de menor número de análises laboratoriais.

Com a presente pesquisa, teve-se como objetivo a determinação da energia metabolizável aparente corrigida (EMAn) de farelos e grãos de soja processados, utilizando um ensaio com frangos em crescimento e equações de predição, fazendo inferências dos valores de EMAn determinados in vivo com aqueles obtidos por equações de predição, publicadas por Janssen et al. (1979), Janssen (1989), Rodrigues (2000) e por Rodrigues et al. (2002), a fim de se verificar a aplicabilidade dessas equações na determinação dos valores energéticos desses alimentos.

\section{MATERIAL E MÉTODOS}

O experimento foi conduzido no Setor de Avicultura do Departamento de Zootecnia - DZO da Universidade Federal de Lavras - UFLA. As aves foram criadas em galpão de alvenaria até a idade de 16 dias, período no qual receberam uma ração inicial para frangos de corte, tendo como ingredientes básicos milho e farelo de soja, formulada de acordo com as recomendações de Rostagno et al. (2000), a qual foi utilizada como raçãoreferência no ensaio de metabolismo (Tabela 1). Após esse período, as aves foram pesadas e transferidas para uma sala de metabolismo com controle de temperatura, recebendo luz artificial por 24 horas.

Foram utilizados 270 pintos da linhagem Cobb, sexados, com peso médio de $447 \mathrm{~g} \pm 5 \mathrm{~g}$, que receberam as rações experimentais constituídas de 8 alimentos e a ração-referência. Os alimentos substituíram a raçãoreferência em $30 \%$, devido ao seu elevado conteúdo de PB. Foi determinada a EMAn de cada alimento testado e da ração-referência em 6 repetições de 5 aves em cada parcela. Determinou-se a EMAn da soja integral extrusada (SIE), soja integral tostada (SIT), soja integral micronizada (SIM) e de 5 amostras de farelos de soja de diferentes marcas comerciais denominados FS1, FS2, FS3, FS4 e FS5.

As rações e água foram fornecidas à vontade, por um período de 10 dias, sendo 7 dias de adaptação (pré-experimental) e 3 dias de coleta total de excretas em cada unidade experimental, a qual foi realizada uma vez ao dia, iniciada sempre às 8 horas da manhã. De acordo com os resultados de Martinez (2002), o período de coleta total de excretas na determinação do valor energético foi reduzido de 5 para 3 dias. Para a coleta das fezes (24 a 26 dias de idade), as bandejas foram revestidas com plástico sob o piso de cada gaiola, para facilitar a coleta e evitar perdas.

O consumo de ração de cada unidade experimental, durante o período de coleta, foi registrado e as excretas coletadas foram colocadas em sacos plásticos devidamente identificados, e armazenadas em freezer. Então, as amostras foram pesadas, homogeneizadas e retiradas as alíquotas devidas para as análises de matéria seca (MS), nitrogênio (N) e energia bruta (EB). Os valores de (EMAn) foram determinados conforme as fórmulas de substituição dos alimentos na ração-referência propostas por Matterson et al. (1965), e ajustados para a retenção de nitrogênio. Para cada alimento, foram determinados: matéria seca (MS), proteína bruta (PB), nitrogênio (N), extrato etéreo (EE), energia bruta (EB), fibra bruta (FB), fibras em detergente ácido e neutro (FDA e FDN), extrativo não-nitrogenado (ENN), matéria mineral (MM), conforme as técnicas descritas por Silva (1990). O amido foi determinado pelo método colorimétrico de Somogy-Nelson, descrito por Nelson (1944). 
TABELA 1 - Composição centesimal e calculada da ração-referência.

\begin{tabular}{|c|c|}
\hline Ingredientes & $(\%)$ \\
\hline Milho & 58,000 \\
\hline Farelo de soja & 35,700 \\
\hline Óleo vegetal & 2,500 \\
\hline Fosfato bicálcico & 1,850 \\
\hline Calcário calcítico & 1,000 \\
\hline Sal & 0,400 \\
\hline DL-Metionina & 0,200 \\
\hline L-Lisina HCL & 0,100 \\
\hline Suplemento vitamínico ${ }^{1}$ & 0,100 \\
\hline Suplemento mineral $^{2}$ & 0,100 \\
\hline Anticoccidiano $^{3}$ & 0,050 \\
\hline TOTAL & 100 \\
\hline \multicolumn{2}{|c|}{ Composição calculada } \\
\hline Energia metabolizável (kcal/kg) & 2970 \\
\hline Proteína bruta (\%) & 21,50 \\
\hline Metionina + cistina (\%) & 0,90 \\
\hline Lisina (\%) & 1,10 \\
\hline Cálcio (\%) & 0,90 \\
\hline Fósforo disponível (\%) & 0,42 \\
\hline Sódio (\%) & 0,20 \\
\hline
\end{tabular}

${ }^{1}$ Contendo por kg do produto: Vit. A - 12.000.000 UI; Vit. $D_{3}$ - 2.200.000 UI; Vit E - 30.000 UI; Vit $B_{1}$ 2,2 g; Vit $B_{2}$ - 6,0 g; Vit $B_{6}-3,3$ g; Vit $B_{12}-0,016$ g; Ácido nicotínico - 53,0 g; Ác. Pantotênico - 13,0 g; Vit. $K_{3}$ - 2,5 g; Ác. Fólico -1,0 g; antioxidante - 120,0 g e veículo q.s.p. - $1000 \mathrm{~g}$.

${ }^{2}$ Contendo por kg do produto: manganês - 75 g; ferro - 20 g; zinco - 50 g; cobre - 4 g; cobalto - 0,2 g; selênio - 0,25 g; iodo - 1,5 g e veículo q.s.p. $-1000 \mathrm{~g}$.

${ }^{3}$ Monensina sódica $20 \%$.

Objetivando comparar os valores de EMAn, estimados pelas equações de predição descritas por Janssen et al. (1979), Janssen (1989), Rodrigues (2000) e Rodrigues et al. (2002), com os valores obtidos diretamente no ensaio metabólico, foram utilizados os dados da composição centesimal dos oito alimentos testados para o cálculo da EMAn. As equações propostas por Rodrigues (2000) e Rodrigues et al. (2002) foram esti- madas com base na composição química e valores de EMAn da soja e farelos de soja, para predizer os valores energéticos desse grupo de alimentos. Porém, as equações de Janssen et al. (1979) e Janssen (1989) são específicas para cada alimento.

Equações de predição (EQP) propostas por Rodrigues (2000) e Rodrigues et al. (2002):

EQP-1

EMAn $=1822,76-99,32 \mathrm{FB}+60,50 \mathrm{EE}+$ 286,73MM - 52,26AMIDO

EQP-2 $\quad$ EMAn $=2822,19-90,13 F B+49,96 E E$

EQP-3 $\quad$ EMAn $=-822,33+69,54 \mathrm{~PB}-45,26 \mathrm{FDA}$ $+90,81 \mathrm{EE}$

EQP-4 $\quad \mathrm{EMAn}=2723,05-50,52 \mathrm{FDA}+60,40 \mathrm{EE}$

Equações de predição (EQP) propostas por Rodrigues (2000):

EQP-5 $\quad$ EMAn $=2372,54+53,69 E E$

EQP-6 $\quad E M A n=7660,52-101,45 P B$

Equações de predição propostas por Janssen et al. (1979):

EQP-8 (para o farelo de soja) $\quad$ EMAn8 $=2702$ $57,4 \mathrm{FB}+72,0 \mathrm{EE}$

EQP-10 (para a soja integral) $\quad$ EMAn10 $=2769$ $59,1 \mathrm{FB}+62,1 \mathrm{EE}$

Equações de predição propostas por Janssen (1989):

EQP-7 (para o farelo de soja) $\quad$ EMAn7 $=37,5 \mathrm{~PB}+$

EQP-9 (para o farelo de soja) $\quad$ EMAn9 $=36,63 \mathrm{~PB}+$

$77,96 \mathrm{EE}+19,87 \mathrm{ENN}$

A fim de se verificar a aplicabilidade das equações citadas, realizou-se uma análise de correlação (Correlações de Spearman), verificando a correlação existente entre os valores energéticos determinados e os valores energéticos estimados por meio das equações de predição e a correlação existente entre as equações. Além da análise de correlação, foram estimados os intervalos de confiança (IC) para as médias dos valores energéticos (EMAn) dos alimentos, obtidos no ensaio metabólico. Os valores calculados pelas equações de predição foram, então, comparados com o IC de cada alimento. As análises estatísticas foram feitas utilizando-se o pacote estatístico SAEG (UFV, 1992), considerando como tratamentos os valores de EMAn determinados no ensaio metabólico e os valores de EMAn estimados pelas equações de predição. 


\section{RESULTADOS E DISCUSSÃO}

A composição química, determinada pelas análises laboratoriais, dos alimentos utilizados no experimento está apresentada na Tabela 2. Os alimentos dentro de seu respectivo grupo, sojas integrais processadas e amostras de farelos de soja, apresentaram diferentes valores nutricionais, quando comparados às tabelas de composição de alimentos internacionais (NRC, 1994; DALE, 2001) e nacionais (EMBRAPA, 1991; ROSTAGNO et al. 2000). Essas diferenças eram esperadas, já que a fertilidade do solo, clima, genética, armazenamento e processamento, principalmente no caso de subprodutos, são fatores que interferem na composição química dos alimentos (ALBINO e SILVA, 1996; BUTOLO, 2002).

Na Tabela 3, estão apresentados os valores energéticos determinados no ensaio metabólico, expressos na matéria seca para comparação com aqueles obtidos na literatura e na matéria natural, sendo essa a forma comumente utilizada na formulação de rações para aves.

TABELA 2 - Composição química de farelos e grãos de soja processados (expressos na matéria natural).

\begin{tabular}{lcccccccc}
\hline \multirow{2}{*}{ Nutriente } & \multicolumn{7}{c}{ Alimento $^{\mathbf{1}}$} \\
\cline { 2 - 8 } & SIE & SIT & SIM & FS 1 & FS 2 & FS 3 & FS 4 & FS 5 \\
\hline $\mathrm{MS}^{2}$ (\%) & 95,21 & 92,64 & 93,99 & 90,47 & 90,69 & 90,70 & 89,77 & 89,49 \\
$\mathrm{~PB}^{2}$ (\%) & 36,24 & 35,15 & 40,99 & 44,30 & 44,24 & 44,09 & 41,62 & 41,76 \\
$\mathrm{~EB}^{2}(\mathrm{kcal} / \mathrm{kg})$ & 4854 & 5254 & 5411 & 4222 & 4217 & 4294 & 4074 & 4079 \\
$\mathrm{EE}^{2}$ (\%) & 22,30 & 22,19 & 24,64 & 3,92 & 2,78 & 3,73 & 4,91 & 5,10 \\
$\mathrm{FDN}^{2}$ (\%) & 12,80 & 18,34 & 12,73 & 11,12 & 12,08 & 9,09 & 13,38 & 13,43 \\
$\mathrm{FDA}^{2}$ (\%) & 8,91 & 9,06 & 3,23 & 10,17 & 9,52 & 7,47 & 11,06 & 10,39 \\
$\mathrm{FB}^{2}$ (\%) & 5,77 & 6,20 & 1,17 & 4,35 & 5,32 & 4,88 & 5,63 & 5,95 \\
$\mathrm{ENN}^{3}$ (\%) & 26,45 & 24,26 & 23,04 & 32,18 & 32,56 & 32,33 & 31,83 & 31,28 \\
$\mathrm{MM}^{2}$ (\%) & 4,46 & 4,84 & 4,15 & 5,73 & 5,79 & 5,67 & 5,79 & 5,40 \\
$\mathrm{Amido}^{4}$ (\%) & 9,00 & 6,93 & 6,24 & 8,49 & 8,57 & 9,90 & 6,49 & 8,86 \\
\hline
\end{tabular}

${ }^{1}$ SIE - soja int. extrusada; SIT - soja int. tostada; SIM - soja int. micronizada; FS- farelos de soja; ${ }^{2}$ Laborátorio de Nutrição Animal/DZO; ${ }^{3}$ Valor calculado; ${ }^{4}$ Laboratório da Ciências dos Alimentos/DCA

TABELA 3 - Valores de energia metabolizável aparente corrigida (EMAn) de farelos e grãos de soja processados, determinados com pintos em crescimento ( 24 a 26 dias de idade) e seus respectivos desvios padrões.

\begin{tabular}{lccc}
\hline \multicolumn{1}{c}{ Alimento } & MS (\%) & EMAn (kcal/kg de MS) & EMAn (kcal/kg de MN) \\
\hline Soja integral extrusada & 95,21 & $3674 \pm 199$ & $3398 \pm 189$ \\
Soja integral tostada & 92,64 & $3609 \pm 228$ & $3343 \pm 212$ \\
Soja integral micronizada & 93,99 & $4296 \pm 207$ & $4025 \pm 194$ \\
Farelo de soja 1 & 90,47 & $2601 \pm 231$ & $2353 \pm 209$ \\
Farelo de soja 2 & 90,69 & $2650 \pm 172$ & $2403 \pm 156$ \\
Farelo de soja 3 & 90,70 & $2727 \pm 238$ & $2473 \pm 216$ \\
Farelo de soja 4 & 89,77 & $2500 \pm 212$ & $2244 \pm 191$ \\
Farelo de soja 5 & 89,49 & $2426 \pm 204$ & $2171 \pm 182$ \\
\hline CV \% & & 6,90 & 7,20 \\
\hline
\end{tabular}

Ciênc. agrotec., Lavras, v. 28, n. 6, p. 1400-1407, nov./dez., 2004 
A EMAn das amostras de farelo de soja variaram de 2426 a $2727 \mathrm{kcal} / \mathrm{kg}$ de MS. As amostras de farelos de soja apresentaram, em média, $2580 \mathrm{kcal}$ de EMAn/kg de MS, valor superior aos encontrados na literatura nacional (EMBRAPA, 1991; CAFÉ, 1993; ROSTAGNO et al., 2000; RODRIGUES et al., 2002) e nas tabelas internacionais (JANSSEN, 1989; NRC, 1994; DALE, 2001).

O valor energético da SIM foi superior em comparação às demais sojas integrais; no entanto, esse valor (4296 kcal de EMAn/kg de MS) foi semelhante aos valores encontrados por Café (1993) e Rodrigues et al. (2002), (4305, 4104kcal de EMAn/kg de MS, respectivamente). De todas as amostras de alimentos avaliadas no presente trabalho, a SIM apresentou maior conteúdo em energia bruta (5411 kcal/kg) e menor teor de fibra bruta $(1,17 \%)$, fato esse que pode ter contribuído para a maior energia metabolizável apresentada, além de que, durante o processo de micronização, ocorre a remoção da casca (JORGE NETO, 1992), melhorando a digestibilidade, o que, aliado à menor granulometria, possibilita uma maior exposição dos nutrientes às enzimas digestivas (CAFÉ, 1993; SAKOMURA, 1996).

Comparando com os valores da literatura, podese observar que o valor de EMAn determinado para a SIE (3674 kcal/kg de MS) foi inferior em 15\% ao valor médio (4324 kcal/kg de MS) apresentado por Bianchi (1996) e próximo (3630 kcal/kg de MS) ao apresentado por Café (1993), usando a metodologia de coleta total com frangos de corte em crescimento.
O valor de $3609 \mathrm{kcal}$ de EMAn/kg de MS para a SIT determinado no presente estudo foi inferior ao valor encontrado por Bianchi (1996), que foi de 3764 E$\mathrm{MAn} / \mathrm{kg}$ de MS, e superior aos valores apresentados por Embrapa (1991), Albino et al. (1992), Café (1993) e Rodrigues et al. (2002), que foram respectivamente 3280, 3467, 3383, 3400 de EMAn/kg de MS. Os valores de EMAn da SIT foram os que apresentaram maior variação na literatura consultada, provavelmente por existir uma grande variação nos tipos de processamentos utilizados.

Na Tabela 4 estão apresentados os resultados das Correlações de Spearman entre a média dos valores de EMAn determinados no ensaio metabólico com a média da EMAn estimada por meio das equações de predição.

Analisando os alimentos em conjunto, observa-se que as equações 3 (EMAn $=-822,33+69,54 \mathrm{~PB}$ - 45,26FDA + 90,81EE); 2 (EMAn = 2822,19 90,13FB + 49,96EE); 7 (EMAn = 1822,76 - 99,32FB $+37,5 \mathrm{~PB}+46,39 \mathrm{EE}+14,9 \mathrm{ENN}) ; 1$ (EMAn = $60,50 \mathrm{EE}+286,73 \mathrm{MM}-52,26 \mathrm{AMIDO})$ e 4 (EMAn = 2723,05 - 50,52FDA + 60,40EE), respectivamente, seguidas da equação 10 (EMAn = $2769-59,1 \mathrm{FB}+$ $62,1 \mathrm{EE})$, associaram-se significativamente $(\mathrm{P}<0,05)$ com o valor médio de EMAn determinado no ensaio metabólico, tendo uma correlação positiva de $85,71 \%$; 80,95\%; 78,57\%; 73,81\%; 73,81 e 64,29\%, respectivamente, mostrando-se boas preditoras dos valores energéticos, confirmando a indicação dos referidos autores.

TABELA 4 - Correlações de Spearman entre a média dos valores de EMAn determinado (ensaio metabólico) com a média da EMAn estimada (equações de predição).

\begin{tabular}{llcccc}
\hline \multicolumn{1}{c}{ Variável } & \multicolumn{1}{c}{ Variável $^{\mathbf{1}}$} & Obser & Correlação & $\mathbf{Z}$ & Signif \\
\hline Ensaio metabólico & Equação 1* $^{*}$ & 8 & 0,7381 & 1,9528 & 0,0254 \\
Ensaio metabólico & Equação 2* $^{*}$ & 8 & 0,8095 & 2,1418 & 0,0161 \\
Ensaio metabólico & Equação 3* $^{*}$ & 8 & 0,8571 & 2,2678 & 0,0117 \\
Ensaio metabólico & Equação 4* & 8 & 0,7381 & 1,9528 & 0,0254 \\
Ensaio metabólico & Equação 5 & 8 & 0,5238 & 1,3859 & 0,0829 \\
Ensaio metabólico & Equação 6 & 8 & 0,5476 & 1,4489 & 0,0737 \\
Ensaio metabólico & Equação 7* & 8 & 0,7857 & 2,0788 & 0,0188 \\
Ensaio metabólico & Equação 8** & 8 & 0,6190 & 1,6378 & 0,0507 \\
Ensaio metabólico & Equação 9 & 8 & 0,5476 & 1,4489 & 0,0737 \\
Ensaio metabólico & Equação 10* & 8 & 0,6429 & 1,7008 & 0,0445 \\
\hline
\end{tabular}

${ }^{1}$ Equações 1 a 4 Rodrigues (2000) e Rodrigues et al. (2002); Equações 5 e 6 Rodrigues (2000); Equações 8 e 10 Janssen et al. (1979); Equações 7 e 9 Janssen (1989)

*Correlação significativa $(\mathbf{P}<\mathbf{0 , 0 5})$; ${ }^{* *}$ Correlação significativa $(\mathbf{P}=\mathbf{0 , 0 5 1})$ 
Observou-se que as equações com duas e quatro variáveis, consideradas no modelo de predição, apresentaram os valores de EMAn calculados mais correlacionados com o valor médio determinado, confirmando as colocações de Rodrigues et al. (2002), os quais observaram que equações com duas a quatro variáveis predizem melhor os valores energéticos. Porém, nem todas equações com esse número de variáveis fazem boas estimativas, pois, apesar de a variável compor a equação, ela deve estar correlacionada com os valores energéticos (RODRIGUES et al., 2002).

Os valores de EMAn dos alimentos, determinados no ensaio in vivo, seus respectivos intervalos de confiança (IC), bem como os valores estimados pelas equações de predição estão descritos na Tabela 5 .

Em nenhuma das equações estudadas foram estimados todos os valores de EMAn dos alimentos dentro do IC calculado.

As equações em que se estimou um maior número de valores energéticos, sete dos oito alimentos avaliados, foram as equações 3 (EMAn $=-822,33+69,54 \mathrm{~PB}$ - 45,26FDA + 90,81EE) e 4 (EMAn = 2723,05 50,52FDA + 60,40EE), nas quais se utilizaram a fibra em detergente ácido e o extrato etéreo para estimar a EMAn, não estimando apenas a amostra de FS2, provavelmente devido ao baixo teor de extrato etéreo contido nesse farelo.

Apesar de utilizar uma equação específica para cada alimento, a equação 9 (EMAn = 36,63PB + 77,96EE + 19,87ENN), indicada para o farelo de soja (JANSSEN, 1989), estimou seis valores energéticos, sendo a EMAn das três sojas integrais (SIE, SIT e SIM) e três amostras de farelos (FS1, FS2 e FS3) e a equação 10 (EMAn = 2769 - 59,1FB + 62,1EE), apesar de ser específica para soja integral, estimou três amostras de farelos de soja (FS1, FS2 e FS3), como também os valores energéticos de duas sojas integrais (SIE e SIM). Diante disso, a especificidade para alimentos, segundo as equações de Janssen (1989), foi observada para a equação 7 (EMAn = 37,5PB + 46,39EE + 14,9ENN), que apresentou uma correlação de 78,57\% e estimou todas as amostras de farelos de soja, mostrando-se boa preditora (Figura 1). Já a equação 8 (EMAn = 2702-57,4FB $+72,0 \mathrm{EE}$ ) estimou três valores de farelos de soja, reforçando a especificidade dessas equações para o farelo $(\mathrm{P}<0,05)$.

TABELA 5 - Valores energéticos determinados, seus respectivos intervalos de confiança (IC) e os valores energéticos estimados pelas equações de predição.

\begin{tabular}{lcccccccc}
\hline \multicolumn{1}{c}{ Alimento } & SIE & SIT & SIM & FS1 & FS2 & FS3 & FS4 & FS5 \\
\hline EMAn $^{2}$ & 3674 & 3609 & 4296 & 2601 & 2650 & 2727 & 2500 & 2426 \\
\hline IC & $3465 \mathrm{a}$ & $3369^{\mathrm{a}}$ & $4079^{\mathrm{a}}$ & $2359^{\mathrm{a}}$ & $2469 \mathrm{a}$ & $2477^{\mathrm{a}}$ & $2277^{\mathrm{a}}$ & $2212^{\mathrm{a}}$ \\
& 3883 & 3849 & 4513 & 2843 & 2831 & 2977 & 2723 & 2640 \\
\hline CV $^{3}$ & 5,41 & 6,33 & 4,82 & 8,87 & 6,50 & 8,75 & 8,48 & 8,39 \\
\hline EMAn1 & 3486 & 3713 & 4204 & 2934 & 2763 & 2759 & 3001 & 2718 \\
EMAn2 & 3446 & 3416 & 4020 & 2605 & 2447 & 2543 & 2530 & 2507 \\
EMAn3 & 3528 & 3549 & 4366 & 2467 & 2373 & 2559 & 2340 & 2415 \\
EMAn4 & 3665 & 3676 & 4133 & 2417 & 2378 & 2555 & 2431 & 2480 \\
EMAn5 & 3630 & 3659 & 3780 & 2650 & 2537 & 2593 & 2666 & 2678 \\
EMAn6 & 3799 & 3811 & 3338 & 2693 & 2712 & 2729 & 2957 & 2926 \\
EMAn7 & 2928 & 2924 & 3217 & 2567 & 2506 & 2545 & 2520 & 2535 \\
EMAn8 & 4040 & 4043 & 4518 & 2738 & 2586 & 2689 & 2736 & 2730 \\
EMAn9 & 3772 & 3778 & 4129 & 2838 & 2739 & 2809 & 2829 & 2848 \\
EMAn10 & 3865 & 3861 & 4324 & 2754 & 2613 & 2706 & 2738 & 2730 \\
\hline
\end{tabular}

${ }^{1}$ Valor energético em itálico está dentro do intervalo de confiança;

${ }^{2}$ Valores energéticos determinados no ensaio de metabolismo expressos em kcal/kg de MS; EMAn-1 a 4 - equações de Rodrigues (2000) e Rodrigues et al. (2002); EMAn- 5 e 6 - equações de Rodrigues (2000); EMAn- 8 e 10 equações de Janssen et al. (1979); EMAn- 7 e 9 - equações de Janssen (1989); ${ }^{3}$ Coeficiente de variação dos IC. 
A equação 1 (EMAn $=1822,76-99,32 \mathrm{FB}+$ 60,50EE + 286,73MM - 52,26AMIDO) apresentou uma correlação positiva (73,81\%) e significativa $(\mathrm{P}<0,05)$, estimando duas amostras de farelos (FS1 e FS2) e as três sojas integrais. No entanto, observa-se que o amido compõe a equação e a sua determinação não é considerada uma análise rotineira em laboratórios, não compondo a análise proximal dos alimentos, o que pode, às vezes, comprometer sua aplicação na predição dos valores energéticos dos alimentos.

\section{CONCLUSÕES}

Os valores energéticos das amostras de farelo de soja variaram de 2426 a $2727 \mathrm{kcal} / \mathrm{kg}$ de MS, sendo mais bem estimados pela equação $\mathbf{E M A n}=\mathbf{3 7 , 5 P B}+$ 46,39EE + 14,9ENN;

Os valores de EMAn das sojas integrais extrusada, tostada e micronizada foram 3674, 3609, 4296 $\mathrm{kcal} / \mathrm{kg}$ de MS, respectivamente, e mais bem estimados pelas equações EMAn $=-\mathbf{8 2 2 , 3 3}+\mathbf{6 9 , 5 4 P B}-$ 45,26FDA + 90,81EE e EMAn = 1822,76 - 99,32FB + 60,50EE + 286,73MM - 52,26AMIDO;

A utilização das equações EMAn $=\mathbf{- 8 2 2 , 3 3 +}$ 69,54PB - 45,46FDA + 90,81EE e EMAn = 2723,05 50,52FDA + 60,40EE para predizer a EMAn da soja integral e farelos de soja é viável.

\section{REFERÊNCIAS BIBLIOGRÁFICAS}

ALBINO, L. F. T.; SILVA, M. A. Valores nutritivos de alimentos para aves e suínos determinados no Brasil. In: SIMPÓSIO INTERNACIONAL SOBRE EXIGÊNCIAS NUTRICIONAIS DE AVES E SUÍNOS, 1996, Viçosa. Anais... Viçosa: UFV, 1996. p. 303-318.

ALBINO, L. F. T. et al. Utilização de diferentes sistemas de avaliação energéticas dos alimentos na formulação de rações para frangos de corte. Revista da Sociedade Brasileira de Zootecnia, Viçosa, v. 21, n. 6, p. 1037-1046, nov./dez. 1992.

BIANCHI, M. del. Efeito da idade do frango de corte na digestibilidade dos nutrientes da soja integral processada pelo calor. 1996. 90 f. Dissertação (Mestrado em Zootecnia) - Universidade Estadual Paulista, Jaboticabal, 1996.

BUTOLO, J. E. Qualidade de ingredientes na alimentação animal. Campinas: [s.n.], 2002. 430 p.
CAFÉ, M. B. et al. Determinação da disponibilidade biológica dos aminoácidos e da energia metabolizável da soja integral processada para aves. In: CONFERÊNCIA APINCO DE CIÊNCIA E TECNOLOGIA AVÍCOLAS, 1993, Santos, SP. Anais... Campinas: FACTA, 1993. p. 13.

DALE, N. Ingredient analysis table: 2001 edition. Feedstuffs, Minneapolis, v. 73, n. 29, p. 220, 2001.

EMPRESA BRASILEIRA DE PESQUISA AGROPECUÁRIA. Centro Nacional de Pesquisa de Suínos e Aves. Tabela de composição química e valores energéticos de alimentos para suínos e aves. 3. ed. Concórdia, 1991. 97 p. (Documentos, 19).

JANSSEN, W. M. M. A. European table of energy values for poultry feedstuffs. 3. ed. Beekbergen: [s.n.], 1989. $84 \mathrm{p}$.

JANSSEN, W. M. M. A. et al. Feeding values for poultry. Cambridge: Cambridge University, 1979.

JORGE NETO, G. Soja integral na alimentação de aves e suínos. Avicultura e Suinocultura Industrial, Porto Feliz, ano 82, n. 988, p. 4-15, jun. 1992.

MARTINEZ, R. S. Avaliação da metodologia e do período de coleta na determinação do valor energético de rações para aves. 2002. 41 p. Dissertação (Mestrado) - Universidade Federal de Lavras, Lavras, 2002.

MATTERSON, L. D. et al. The metabolizable energy of feed ingredients for chickens. Storrs: The University of Connecticut, 1965. 11 p. (Research Report, 7).

NATIONAL RESEARCH COUNCIL. Nutrient requirements of poultry. 9. ed. Washington: National Academy, 1994. 155 p.

NELSON, N. A. A photometric adaptation of Somogy method for the determination of glucose. Journal of Biological Chemistry, Baltimore, v. 153, p. 375-380, 1944.

RODRIGUES, P. B. Digestibilidade de nutrientes e valores energéticos de alguns alimentos para aves. 2000. 204 f. Tese (Doutorado em Zootecnia) - Universidade Federal de Viçosa, Viçosa, 2000.

RODRIGUES, P. B. et al. Valores energéticos da soja e subprodutos da soja, determinados com frangos de corte e galos adultos. Revista Brasileira de Zootecnia, Viçosa, v. 31, n. 4, p. 1771-1782, 2002. 
ROSTAGNO, H. S. et al. Tabelas brasileiras para aves e suínos: composição de alimentos e exigências nutricionais. Viçosa: UFV, 2000. 141 p.

SAKOMURA, N. K. Estudo do valor nutricional das sojas integrais processadas e de sua utilização na alimentação de frangos e poedeiras. 1996. $178 \mathrm{f}$. Tese (Livre Docência em Avicultura) - Universidade Estadual Paulista, Jaboticabal, 1996.
SILVA, D. J. Análise de alimentos: métodos químicos e biológicos. 2. ed. Viçosa: UFV, 1990. $165 \mathrm{p}$.

UNIVERSIDADE FEDERAL DE VIÇOSA. SAEG - Sistema de Análises Estatísticas e Genéticas: manual do usuário. versão 5.0. Viçosa: UFV, 1992. 59 p. 